\title{
Fixation biologique d'azote et évolution des marges glaciaires de haute altitude.
}

\author{
Biological nitrogen fixation and the \\ development of glacial margins at high altitude
}

\author{
A. Moiroud \\ Département de biologie végétale, \\ Université Lyon I
}

Les marges glaciaires, c'est-à-dire cette zone située à l'aval du front d'un glacier et qui est progressivement découverte par le retrait de la glace, constituent un milieu neuf qui sera progressivement colonisé et transformé par les agents biotiques. Le lithosol d'érosion ainsi libéré présente deux caractéristiques essentielles :

- il est soumis à l'action d'un facteur climatique prépondérant, la température,

- il est très pauvre en certains éléments essentiels à la vie, comme l'azote par exemple (Moiroud, 1976).

Les faibles teneurs en azote des dépôts morainiques ainsi abandonnés limitent très fortement à la fois l'activité de la microflore et la croissance des végétaux supérieurs (Moiroud, 1976). Aussi, tout apport de cet élément, même faible et localisé, va provoquer des modifications profon- des dans l'activité des microorganismes et le développement des plantes pionnières. La fixation biologique d'azote, par les quantités d'azote qu'elle est susceptible de mettre en jeu, apparaît comme un élément déterminant dans la colonisation des marges glaciaires et leur évolution vers le stade climacique (Crocker et Major, 1955; Paternoster, 1981). Bien que la présence de fixateurs libres (Cyanobactéries) ou symbiotiques (diverses espèces de trèfle, aulne vert) sur les marges glaciaires soit connue depuis longtemps (Moiroud et Gonnet, 1974; Moiroud, 1976) aucune évaluation de la fixation n'avait encore été faite dans ces zones. Nous rapporterons donc dans cette note des résultats concernant des mesures de fixation d'azote effectuées sur diverses marges glaciaires dans les Alpes Occidentales.

\section{Matériel et méthodes}

\section{Localisation des stations}

Les mesures de fixation biologique ont surtout été réalisées sur les marges du glacier de Saint-Sorlin (Savoie) et du glacier de la Pilatte (Isère). D'autres mesures, plus rares, ont été effectuées à titre de comparaison sur la marge d'autres glaciers comme le Chardon (Isère), ou le Jandri (Isère).

Les stations ont été choisies dans des zones déglacées depuis quelques années à environ 1 siècle.

\section{Mesure de la fixation d'azote}

Nous avons utilisé la méthode indirecte de réduction de l'acétylène (Stewart et al., 1967). L'activité réductrice d'acétylène (ARA) des fixateurs libres a été mesurée in situ sur des échantillons de sol prélevés soit dans les zones de sol nu soit dans la rhizosphère de diverses plantes pionnières, et enfermés dans des flacons à fermeture hermétique. D'autres mesures ont été réalisées au laboratoire sur des échantillons de sol enrichis ou non par apport d'un milieu minéral dépourvu d'azote et incubés pendant des temps variés et dans différentes conditions de température et d'éclairement. Des essais de dénombrement de fixateurs libres ont également été entrepris.

L'ARA des nodules de trèfle ou d'aulne vert a été mesurée in situ sur des nodules séparés du système racinaire de l'hôte. Les nodules étaient placés dans des flacons à fermeture hermétique maintenus à la température du sol. En outre et pour Trifolium badium uniquement, nous avons effectué des mesures sur des carottes de sol contenant chacune un pied entier de trèfle. Tous les

LA HOUILLE BLANCHE/N ${ }^{\circ}$ 6/7-1984 
échantillons ont été incubés pendant 1 heure dans une atmosphère contenant $10 \%$ de $\mathrm{C}_{2} \mathrm{H}_{2}$. L'ARA de jeunes plants de Trifolium badium, $T$. Thalii, $T$. pallescens et Alnus viridis développés au laboratoire à partir de graines récoltées dans la nature a aussi été mesurée. Les 3 espèces de trèfle utilisées ont été bactérisées avec une suspension de Rhizobium isolées de nodules de $T$. badium récoltés sur la marge du glacier de Saint-Sorlin, vers $2400 \mathrm{~m}$ d'altitude. Les jeunes plants d'aulne vert ont été inoculés avec un broyat de nodules récoltés dans la nature.

\section{Résultats}

\section{Fixation libre}

Il ne nous a jamais été possible de mettre en évidence in situ la moindre activité réductrice de la part d'organismes fixateurs libres. De même nous n'avons jamais pu isoler de bactéries fixatrices libres d'azote à partir des lithosols glaciaires, quels que soit les techniques ou les milieux utilisés. Par contre nous avons toujours pu mettre en évidence la présence, après saturation des échantillons de sol et incubation pendant plusieurs semaines à $22^{\circ} \mathrm{C}$, de Cyanobactéries dans les divers échantillons de sol étudiés (Tab. I).

\begin{tabular}{|l}
\begin{tabular}{|l} 
Tabl. I.- Activité réductrice d'acétylène exprimée en $\mu \mathrm{I}$ \\
de $\mathrm{C}_{2} \mathrm{H}_{4} / \mathrm{h} /$ flacon de divers échantillons de sol mesurée
\end{tabular} \\
dès leur prélèvement sur le terrain et après incubation \\
pendant 8 semaines au laboratoire en conditions opti- \\
males (To = $22{ }^{\circ} \mathrm{C}$, éclairement 4000 lux, sol saturé avant \\
incubation par une solution minérale dépourvue d'azote).
\end{tabular}

Les Cyanobactéries développées sur les différents échantillons de sol appartiennent essentiellement à la famille des Nostocacées. Parmi les formes morphologiques rencontrées certaines ont pu être rapportées aux genres Nostoc et Anabaena alors que les autres n'ont pas pu être déterminées avec certitude.

La plupart de ces Cyanobactéries montraient la présence d'hétérocystes. L'utilisation de la méthode de réduction de l'acétylène a du reste permis de vérifier leur aptitude à fixer $\mathrm{l}^{\prime} \mathrm{N}_{2}$ atmosphérique. L'ARA croît avec la température (fig. 1); elle présente un optimum net vers $26^{\circ}$ et décline rapidement au delà.

Elle est encore mesurable à des températures voisines de $4{ }^{\circ} \mathrm{C}$ (fig. 1). Il est du reste remarquable que les Cyanobactéries des marges glaciaires conservent leur aptitude à réduire l'acétylène pendant plusieurs mois à $4^{\circ} \mathrm{C}$, à condition toutefois qu'elles se soient préalablement développées à $22^{\circ} \mathrm{C}$. En effet ces Cyanobactéries sont incapables de croître à des températures inférieures à $10^{\circ} \mathrm{C}$. L'ARA varie également avec l'intensité de l'éclairement; elle est très réduite pour de faibles éclairements et atteint son maximum aux environs de 3000 lux. Les éclairements supérieurs sont sans effet.

\section{Fixation symbiotique}

\section{1. fixation par les trèfles}

Nous avons toujours pu mesurer une activité réductrice de $\mathrm{C}_{2} \mathrm{H}_{2}$ importante in situ soit sur des plants entiers de Trifolium soit sur des nodules excisés (Tabl. II). Du reste tous les pieds de trèfle développés sur les marges glaciaires sont toujours porteurs de nodules et ce quelle qu'en soit l'espèce. Ceci n'est pas surprenant car les essais de dénombrement nous ont montré que, dans les zones oủ les trèfles étaient présents, on trouvait environ $500000 \mathrm{Rhi}$ zobium libres par gramme de sol sec.

\begin{tabular}{|c|c|}
\hline Plantes entières & Nodules excisés \\
\hline $\begin{array}{l}4,5 \mu \mathrm{gN} / \mathrm{h} / \text { plante } \\
9,2 \mu \mathrm{gN} / \mathrm{h} / \text { plante } \\
4,5 \mu \mathrm{gN} / \mathrm{h} / \text { plante }\end{array}$ & $\begin{array}{l}280 \mu \mathrm{gN} / \mathrm{h} / \mathrm{g} \text { nodule sec } \\
226 \mu \mathrm{gN} / \mathrm{h} / \mathrm{g} \text { nodule sec }\end{array}$ \\
\hline \multicolumn{2}{|c|}{$\begin{array}{l}\text { plantes entières: moyenne établie sur } 6 \text { plantes pour } \\
\text { chaque détermination. } \\
\text { nodules excisés : moyenne de } 3 \text { répétitions } \\
\text { Le facteur de conversion } \mathrm{C}_{2} \mathrm{H}_{4}-\mathrm{N} \text { a été déterminé par } \\
\text { utilisation de }{ }^{15} \mathrm{~N}_{2} \text {. }\end{array}$} \\
\hline
\end{tabular}

Au laboratoire, nous avons pu constater que les processus d'infection et de nodulation de jeunes plants de $T$. badium, $T$. pallescens ou $T$. Thalii sont très rapides puisque 10 jours environ après la bactérisation les premiers nodules sont observables. Toutefois ce n'est que lorsque les plantules ont atteint le stade 2 à 3 feuilles trifoliolées que les nodules contiennent de la léghémoglobine et deviennent efficients. A ce stade le nombre de nodules est le plus souvent compris entre 6 et 15 . L'ARA s'élève rapidement avec le développement de la plante et atteint une valeur de l'ordre de $5 \mu$ Moles de $\mathrm{C}_{2} \mathrm{H}_{4} /$ plant/ heure 90 jours après que les nodules soient devenus efficients (fig. 2).

Par des mesures de biomasse et des dosages d'azote il est aussi possible d'estimer les quantités d'azote fixé au cours de la croissance des plantes car dans nos conditions expérimentales la totalité de leur azote a pour origine l' $\mathrm{N}_{2}$ atmosphérique. Les résultats obtenus montrent que les quantités moyennes d' $\mathrm{N}_{2}$ fixé par des plants de $T$. badium âgés de 120 jours sont de l'ordre de $15 \mathrm{mg}$.

Leur teneur moyenne en $\mathrm{N}$ est de $2,3 \%$ ce qui correspond aussi à la teneur moyenne des plants développés sur le terrain.

L'ARA des jeunes plants paraît très sensible aux variations de température. Ainsi, si on soumet des plants de $T$. badium à un abaissement brutal de température 


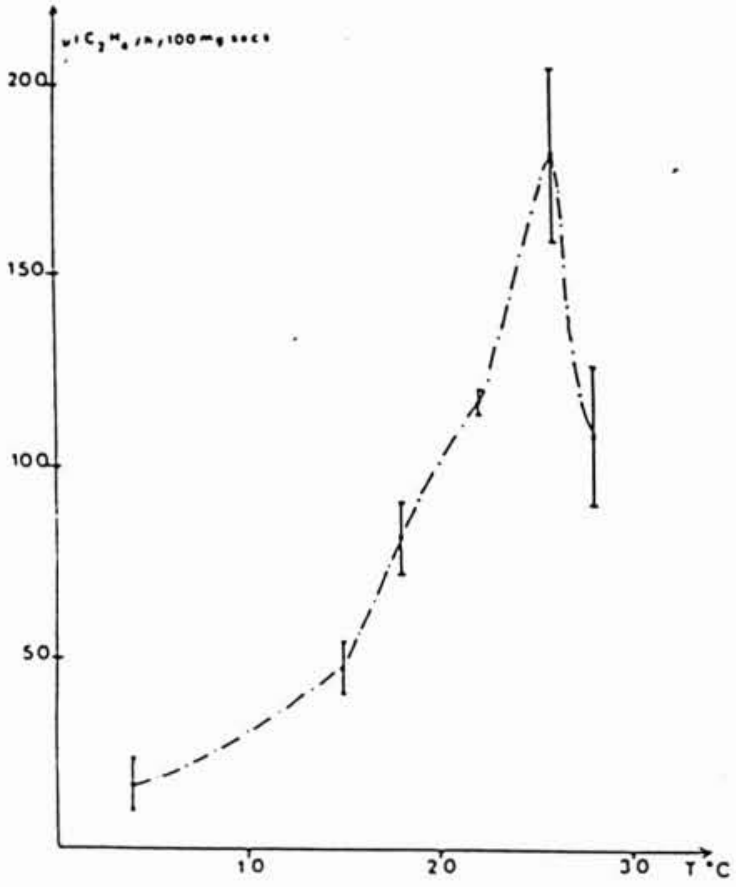

Figure 1 - Activité réductrice d'acétylène (exprimée en $\mu \mathrm{l}$ de $\mathrm{C}_{2} \mathrm{H}_{2} / \mathrm{h} / 100 \mathrm{mg}$ secs) de cultures mixtes de Cyanobactéries à différentes températures.

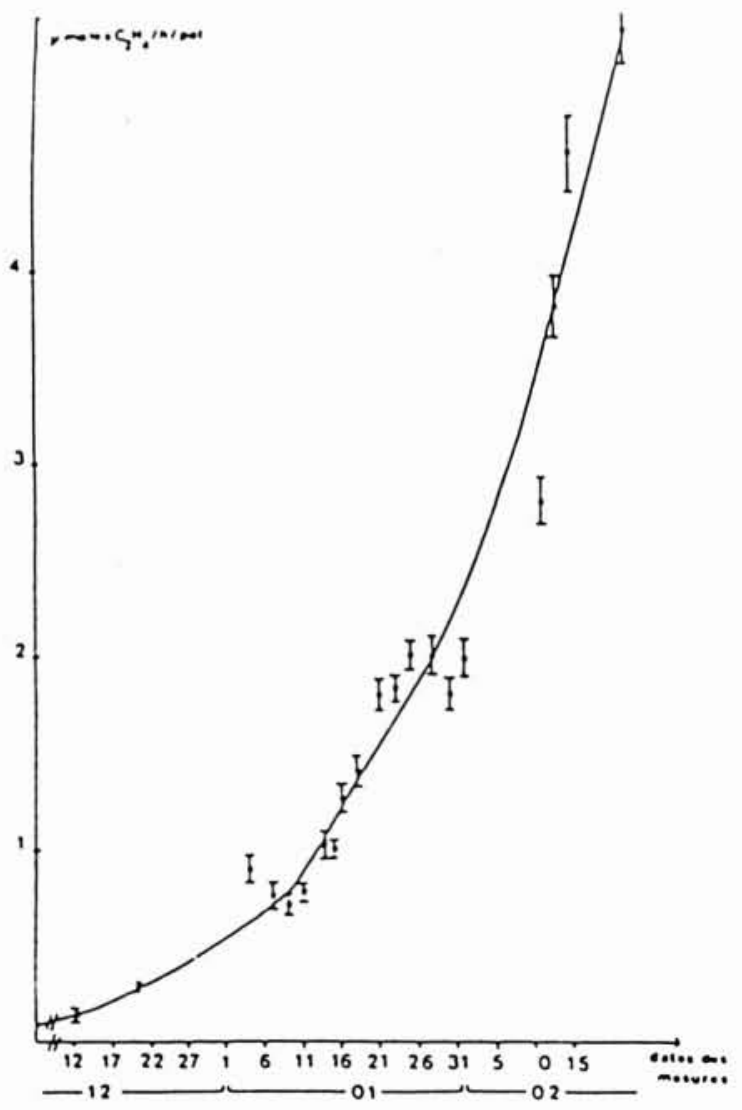

Figure 2 - Activité réductrice d'acétylène de Trifolium badium cultivé à une température alternée de $26^{\circ} \mathrm{C}-16^{\circ} \mathrm{C}$ (moyenne établie sur 10 pieds).

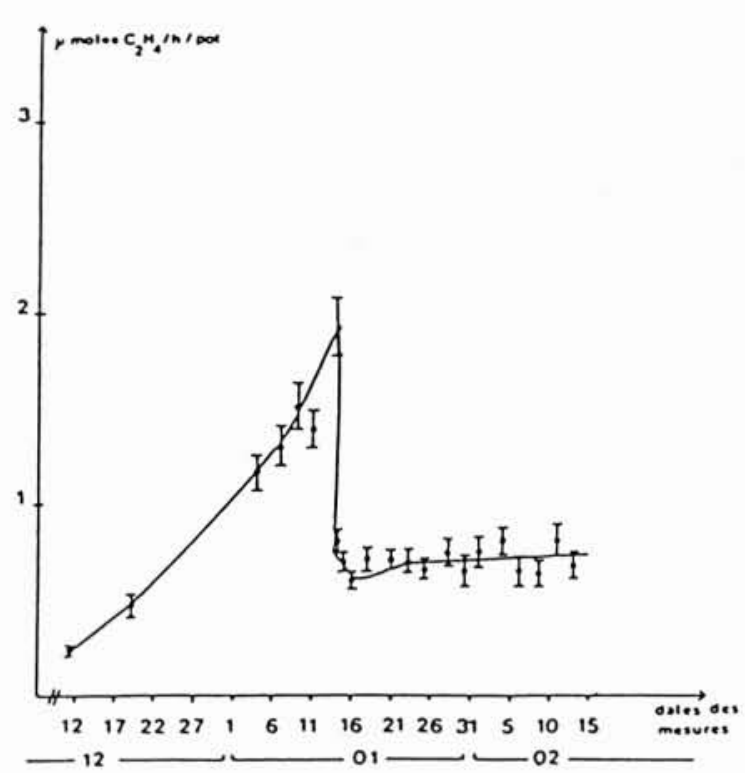

Figure 3 - Activité réductrice d'acétylène de Trifolium badium cultivé ả une température racinaire alternée de $26^{\circ} \mathrm{C}-16^{\circ} \mathrm{C}$ puis placé à une température alternée de $19{ }^{\circ} \mathrm{C}-13^{\circ} \mathrm{C}$ (moyenne établie sur 10 pieds).

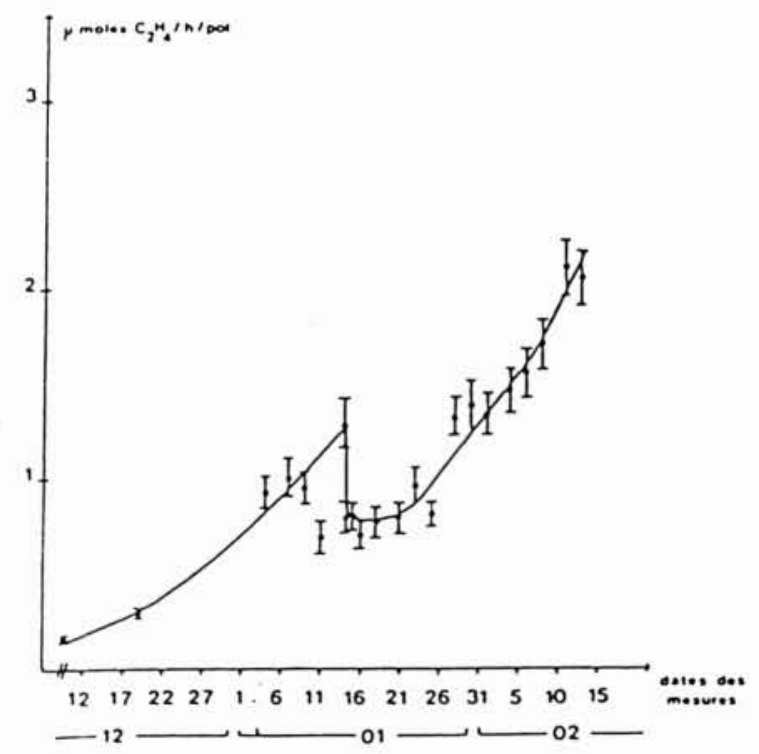

Figure 4 - Activité réductrice d'acétylène de Trifolium badium cultivé à une température racinaire alternée de $26^{\circ} \mathrm{C}-16^{\circ} \mathrm{C}$ puis placé à une température alternée de $14^{\circ} \mathrm{C}-9{ }^{\circ} \mathrm{C}$ (moyenne établie sur 10 pieds). 
(passage d'une température racinaire de $26^{\circ} \mathrm{C}$ le jour et $16^{\circ} \mathrm{C}$ la nuit à une température de $19^{\circ} \mathrm{C}$ le jour et $13^{\circ} \mathrm{C}$ la nuit) on observe une chute rapide de l'ARA (fig. 3 ). Toutefois il est à remarquer que l'ARA augmente de nouveau régulièrement après 12 jours environ de séjour dans ces nouvelles conditions.

Elle ne représente cependant à ce moment que $60 \%$ de l'ARA des plants témoins.

Lorsque l'abaissement de la température racinaire est encore plus prononcé (transfert des plantes d'une alternance $26^{\circ} \mathrm{C}-16^{\circ} \mathrm{C}$ à une alternance $14^{\circ} \mathrm{C}-9^{\circ} \mathrm{C}$ ) on observe une chute spectaculaire de l'ARA, quelques heures seulement après l'application des nouvelles conditions de température (fig. 4). Dans ces conditions, et contrairement à ce qu'on pouvait observer précédemment, l'ARA demeure, pendant toute la durée du séjour à basse température, à un niveau constamment bas qui ne représente qu'environ $8 \%$ de l'activité des témoins. On observe également, chez les plantes maintenues à basse température, une accumulation de sucres hydrosolubles au niveau des racines ( $11 \%$ contre $2,5 \%$ pour les témoins).

Après retour des plants dans les conditions antérieures, l'ARA atteint, après environ 20 jours, une valeur voisine de celle mesurée chez les témoins.

\section{2. fixation par l'aulne vert}

Les mesures effectuées dans la haute vallée du Vénéon montrent que l'activité fixatrice d'azote d'Alnus viridis en pleine période végétative est de l'ordre de $170 \mu \mathrm{gN} / \mathrm{h} / \mathrm{g}$ nodule sec (fig. 5). Cette activité nitrogénasique varie au cours de la journée et augmente avec l'intensité de l'éclairement.L'activité maximum se situe peu de temps après le passage du soleil au zénith.

Les valeurs de fixation obtenues sont du même ordre de grandeur que celles mesurées dans d'autres aulnaies vertes de plus basse altitude (fig. 5). Par contre elle apparaît comme inférieure à celle mesurée pour des nodules de T. badium. Cependant, par suite de la présence

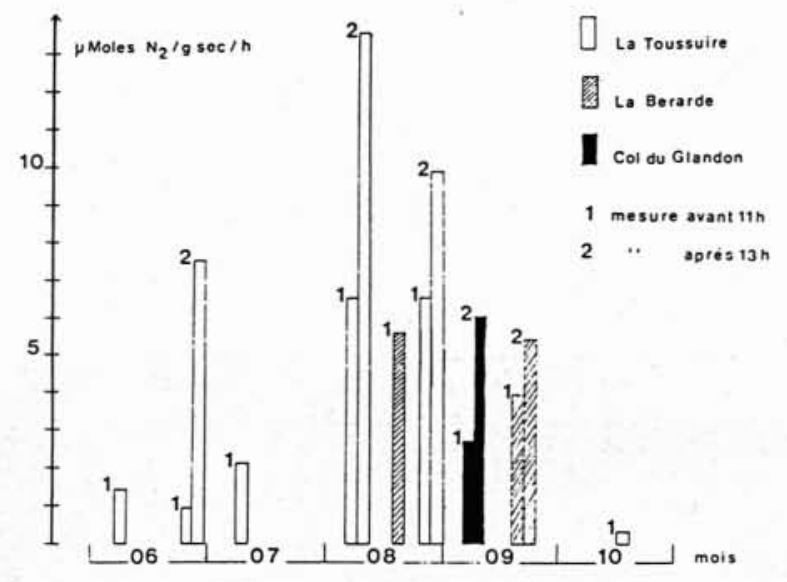

Figure 5 - Activité fixatrice d'azote de nodules excisés d'Alnus viridis au cours de la période végétative. d'une biomasse nodulaire importante - évaluée à $40 \mathrm{~kg} / \mathrm{ha}$ dans la haute vallée du Vénéon - les quantités totales d'azote fixé sont importantes. On peut les estimer à environ $3 \mathrm{~kg} / \mathrm{ha}$ pour les mois de juillet et août.

\section{Discussion}

Les apports d'azote par les bactéries fixatrices libres peuvent être considérés comme nuls sur les marges glaciaires. Ceci s'explique facilement lorsqu'on sait que la réduction de l' $\mathrm{N}_{2}$ atmosphérique en $\mathrm{NH}_{3}$ est un processus coûteux en énergie et que les lithosols d'érosion sont très pauvres en substrats énergétiques, même au niveau de la rhizosphère des plantes pionnières (Moiroud, 1976; Paternoster, 1981). Des résultats similaires avaient déjà été obtenus par d'autres auteurs pour de nombreux autres sols froids (Alexander, 1974; Jordan et al., 1978; Tosca et Labroue, 1981).

Les Cyanobactéries, elles non plus, ne paraissent pas capables d'augmenter sensiblement le stock d'azote des limons morainiques. Bien que toujours présentes dans les sols glaciaires, elles ne constituent cependant jamais des populations importantes, même dans des dépressions humides. Du reste, leur mise en évidence nécessite une incubation des échantillons de sol de plusieurs semaines dans des conditions optimales d'environnement. Sur le terrain nous n'avons jamais observé de Cyanobactéries ni dans des croûtes algales, ni dans des communautés de mousses, ni comme symbiotes de lichens à la différence des écosystèmes arctiques (Shell et Alexander, 1973; Alexander, 1974; Basilier et Granhall, 1978). De plus certaines conditions d'environnement régnant à haute altitude comme la forte irradiation solaire, les grandes amplitudes thermiques et la dessiccation parfois très poussée des lithosols glaciaires sont connues pour avoir une action fortement inhibitrice sur l'activité fixatrice des Cyanobactéries (Alexander, 1974).

La fixation symbiotique d'azote apparait comme le seul processus capable d'augmenter de façon notable le stock d'azote des lithosols glaciaires. Toutefois ces gains en azote ne sont pas toujours faciles à déterminer avec précision à haute altitude par suite de la très grande hétérogénéité dans la répartition de la végétation et des fluctuations parfois très rapides des conditions d'environnement (Tosca et Labroue, 1981).

Les résultats obtenus au laboratoire et les mesures sur le terrain montrent que les plantes fixatrices symbiotiques d'azote sont capables de tirer, in situ tout l'azote nécessaire à leur développement de l'azote atmosphérique. Cette capacité qu'ont ces espèces symbiotiques de croître sur des sols très pauvres en azote et de conserver une activité fixatrice non négligeable dans des conditions défavorables, jointe à des possibilités importantes de multiplication font que ces plantes constituent souvent la part la plus importante de la biomasse végétale sur les lithosols morainiques. Ainsi, sur la marge du glacier de Saint-Sorlin la biomasse sur pied du groupement dominé par Trifolium Thalii est environ 5 fois supérieure à celle des zones 
pionnières sans trèfle (Moiroud, 1976). Sur d'autres marges glaciaires de plus basse altitude (glacier d'Aletsch, glaciers de la haute vallée du Vénéon par exemple) où se rencontrent des peuplement purs d'aulnes verts, cette biomasse représente plusieurs tonnes /ha.

Par apport au sol d'une litière abondante (évaluée à 2 tonnes/ha/an pour des aulnaies vertes de la haute vallée du Vénéon, ce qui correspond à un apport annuel de $45 \mathrm{~kg}$ d'azote organique/ha), les plantes fixatrices enrichissent notablement le sol en matière organique. Grâce à leur forte teneur en azote (environ $2,5 \% \mathrm{~N} / \mathrm{g} \mathrm{sec}$ ) et leur $\mathrm{C} / \mathrm{N}$ bas (12 pour $T$. Thalii, contre 27 pour Leucanthemum alpinum et 32 pour Carex curvula; 18 à 20 pour $A$. viridis) ces débris organiques sont rapidement dégradés et les composés minéraux immobilisés libérés. L'activité de la microflore tellurique est également supérieure dans la rhizosphère des plantes fixatrices symbiotiques (Moiroud, 1976). Ces plantes fixatrices vont donc jouer un rôle important dans l'initiation et le développement des processus pédogénétiques. Ainsi après la mise en place d'un stade à fixateurs symbiotiques d'azote, l'évolution de la végétation et la pédogénèse sur les marges glaciaires deviennent beaucoup plus rapides (Crocker et Major, 1955; Paternoster, 1981).

Cette importance prépondérante des fixateurs symbiotiques d'azote par rapport aux autres types de fixateurs distingue aussi nettement les zones alpines des autres zones froides du globe (Alexander et Schell, 1973).

Conclusion

Les résultats obtenus montrent que différents types de fixateurs d'azote sont présents sur les marges de quelques grands glaciers des Alpes Occidentales. Toutefois le rôle de ces fixateurs dans le gain en azote au niveau du sol est très inégal. Ainsi les fixateurs libres, représentés uniquement par des Cyanobactéries, semblent n'avoir aucune importance réelle. Par contre les fixateurs symbiotiques, représentés surtout par des espèces de trèfle et par l'aulne vert sont, eux, capables d'enrichir de façon importante les lithosols glaciaires en azote. Par là même ils jouent un rôle essentiel dans le dynamisme de la végétation et la mise en place d'un véritable sol.

\section{Références bibliographiques}

ALEXANDER V., 1974. - A synthesis of the IBP Tundra Biome Circumpolar study of nitrogen fixation. In : Holding A.J. ed. : Soil organisms and decomposition in Tundra. Tundra Biome Sterring Committee, Stockholm, p. 109-121.

ALEXANDER V., SCHELL D.M., 1973. - Seasonal and spatial variation of nitrogen fixation in the Barrow, Alaska, Tundra. Arct. Alp. Res., 5; 77-88.

BASILIER K., GRANHALL U., 1978. - Nitrogen fixation in wet minerotrophic moss communities of a subarctic mire. Oikos, 31; 236-246.
CROCKER R.T., MAJOR J., 1955, - Soil development in relation to vegetation and surface age at Glacier Bay, Alaska. J. Ecol., 43; 427-448.

JORDAN D.C., Mc NICOL P.J., MARSHALL M.R., 1978. Biological nitrogen fixation in the terrestrial environment of a high arctic ecosystem. Can. J. Microbiol., 24; 643-649.

MOIROUD A. 1976. - Etude écologique des marges glaciaires, en particulier de leur micropeuplement (exemple du glacier de Saint-Sorlin, Savoie). Thèse Doc. Sci. Lyon 1, 168 p.

MOIROUD A., GONNET J.F., 1974. - Les premières étapes de la colonisation végétale de la moraine du glacier de Saint-Sorlin. Bull. Soc, Linn. Lyon, 10; 387-394.

PATERNOSTER M., 1981. - Colonisation par la végétation et pédogénèse initiale sur les moraines latérales historiques $d u$ grand glacier d'Aletsch. Thèse $3^{\circ}$ Cycle, Nancy, $106 \mathrm{p}$.

SCHELL D.M., ALEXANDER V., 1973. - Nitrogen fixation in Arctic Coastal Tundra in relation to vegetation and microrelief. Arctic 26; 130-137.

STEWART W.D.P., FITZGERALD G.P., BURRIS R.H., 1967. In situ studies on $\mathrm{N}_{2}$ fixation using the acetylene reduction technique. Proc. Natl. Acad. Sci. USA, 58; 2071-2078.

TOSCA C., LABROUE L., 1981. - Le cycle de lazote dans les milieux supraforestiers des Pyrénées Centrales : contribution à l'évaluation des gains. Oecol. Plant., 2; 41-52.

\section{Discussion}

Président : M. L. de CRÉCY

\section{M. le Président remercie Monsieur A. Molroud.}

M. PONCET. - L'exposé de Monsieur A. Molroud sur la pédogénèse en haute altitude à partir d'un substrat purement minéral est très intéressant dans l'optique de la R.T.M. Vous nous avez présenté une vue du versant du Carrelet près de la Bérarde (Vénéon, Oisans) pour illustrer l'influence des aunes verts sur l'enrichissement du sol en azote. II y avait sur le cliché bon nombre de pins à crochets. Avez-vous étudié les propriétés de cet arbre du point de vue de l'enrichissement du sol en azote, car il a la réputation de fixer l'azote d'origine atmosphérique grâce à des mycorhizes ?

M. MoIroud. - Cette idée est abandonnée. La fixation de l'azote ne relevait que des bactéries associées aux champignons. Celle-ci ne doit pas jouer de façon appréciable. Les pins et épicéas vivant à proximité d'aunes verts poussent bien verts et ont en général des teneurs en azote supérieures.

M. PONCET fait remarquer que ce résineux a des exigences pédologiques réduites. En Briançonnais, en Haute Maurienne et Ubaye, il pousse sur des éboulis et même sur le gypse saccharoïde pur et arrive à y constituer, au profit d'autres espèces exigeantes en azote (épicéas) de bons sols forestiers. L'exemple des reboisements d'altitude effectués en épicéa commun à Saint-Colombanles-Villards (Savoie) illustre la valeur pédologique des aunes verts. Dans les alpages abandonnés, les plants d'épicéas sont souffreteux et jaunâtres (manque d'azote). Dans l'aunaie verte (arkoses) les jeunes épicéas sont vigoureux et verts, sous réserve de dégagement des branches feuillues qui peuvent les étouffer.

M. Molroud. - La vallée du Vénéon (Oisans) offre un bel exemple de stades sans aunes, de colonisation et d'aunaies bien développées. Un sol avec litière organique se constitue, avec un début de migration en profondeur.

M. PONCET retient les vertus fixatrices d'azote du trêfle bai sur les moraines glaciaires, témoignage des vertus pédogénétiques des papillonacées. 
M. BAZIRE. - Les apports atmosphériques d'azote ne sont pas négligeables. Ils sont utilisables par les mycorhizes, ce qui peut expliquer le développement des pins à crochets sur des sols squelettiques très pauvres en l'absence d'aunes verts et d'autres plantes fixatrices d'azote.

M. MoIRoud. - On imagine mal qu'en haute altitude les arbres puissent pousser sans mycorhizes.

$M^{m+e}$ EVIN. - L'aune vert et les trèfles sont des plantes colonisatrices. Peut-on proposer un âge pour l'apparition des trèfles?

M. MOIROUD. - Les trèfles se trouvent dans des zones proches de la glace. Sur les marges du glacier de Saint-Sorlin, on le rencontre déjà dans les zones déglacées depuis 10 ou 15 ans. Le problème est de savoir qui viendra en premier : la plante ou la bactérie ? Dans les marges du glacier de Saint Sorlin, non encore colonisées par les trèfles, les bactéries symbiotiques du trèfle existent déjà.

\section{LLIBOUTRY. - Le trèfle ne vient pas le premier.}

M. MOIROUD. - $\mathrm{Il}$ vient relativement tôt. Nous avons vérifié en laboratoire que, sur sol très pauvre, une plante pionnière vit presque en circuit fermé (la deuxième feuille se développe quand la première meurt). Le système racinaire très développé permet d'explorer un grand volume de sol. En outre, des apports peuvent intervenir par les précipitations et l'eau de ruissellement.

Monsieur le Président. - Quelles sont les perspectives de la biotechnologie en matière de production d'azote (engrais azotés...).

M. MOIROUD. - Les forestiers pensent à la fixation d'azote pour améliorer les apports d'azote dans les écosystèmes. On imagine aussi de rendre fixatrices des plantes qui ne le sont pas, des céréales par exemple, en introduisant dans leur génome les gènes de la fixation. Mais il s'agit là de perspectives à long terme !

Présentation du film : Les laves torrentielles à la fonte des neiges à la Ravoire, Bourg-Saint-Maurice (31 mars 1981, CEMAGREF de Grenoble)

\section{Discussion}

Président : M. L. de Crécy

Le Président rappelle que l'intérêt majeur du film est d'avoir pu saisir sur le vif une véritable lave torrentielle. L'accident de la Ravoire a duré trois jours. Toutes les vingt minutes environ se produisait une petite embâcle et une accumulation de matériaux à l'origine de la lave torrentielle. Grâce au pompage de l'eau vers la conduite EDF de Malgovert, l'alimentation en eau a pu être stoppée et le phénomène s'est arrêté. Cet accident s'est produit sur un torrent qui ne s'était jamais encore signalé par des laves torrentielles. En 1967-68, l'urbanisation d'une partie de la région (création de la station des Arcs) a provoqué l'imperméabilisation d'une partie du bassin-versant. Les routes ont contribué à la concentration de l'écoulement, cela peut suffire à doubler le débit de pointe. Le bassin-versant est constitué de schistes houillers altérés; le pavage naturel du lit des petits ruisseaux a été destabilisé. Le creusement s'est accentué par autocatalyse. Les signes de déséquilibre sont apparus en 1970-73 et 74, dates des premières laves torrentielles.

M. PONCET fait remarquer qu'à l'imperméabilisation liée à l'urbanisation doivent s'ajouter les effets du déboisement: déboisements autorisés pour l'ouverture de pistes de ski dans la forêt communale soumise au régime forestier (épicéas, mélézes); défrichements d'anciens alpages recolonisés spontanément. Il faut aussi tenir compte de la destruction des sols forestiers lors de l'amélioration du profil des pistes (diminution de la capacité de rétention dans le bassin-versant).

M. Le Président: Aucune information précise sur le déboisement n'a pu être fournie. Le chiffre de 25 ha concerne des alpages reboisés naturellement et non comptabilisés comme forêts. L'influence est donc sans doute réelle.

$M$. de BEAUREGARD: Quel a été le rôle des pluies ?

Monsieur le Président: Il y a eu conjonction de la fonte des neiges (31 mars) liée à un fort réchauffement et de pluies abondantes.

M. LliboutRY: Dans les pays en voie de développement, au Pérou par exemple, les déboisements ont été intenses en raison des besoins et ont engendrés des laves torrentielles catastrophiques.

M. ROBERT évoque les crues extraordinaires de 1938 en Andorre qui provoquaient des entraînements, très impressionnants, dans le lit de gros blocs de pierre. Il évoque aussi les crues catastrophiques de 1940 dans les Pyrénées Orientales et notamment dans la vallée du Tech, la formation d'un barrage provisoire dans le lit à la suite de l'éboulement des versants. La rupture de ce barrage entraîna des dégâts considérables à l'aval.

M. SOUTADE : Que pensez-vous de la gestion forestière dans ces grands bassins versants de l'Isère, en pente forte, à formations superficielles épaisses et à substrat fragilisé sur lesquels se développe une forêt impressionnante. Que doit-on penser de la gestion forestière?

M. PONCET: Si la forêt protège les versants contre l'érosion superficielle, son action stabilisatrice en profondeur est moins évidente et parfois contestée. Certains ont reproché aux forêts, en favorisant linfiltration des eaux météoriques, de permettre le déclenchement des glissements de terrain. L'argument, valable dans certains cas particuliers, est contredit par l'analyse statistique de la localisation des glissements de terrain par rapport à la nature du couvert végétal. Le rôle de l'évapotranspiration et le développement racinaire contribuent à protéger les terrains exposés à la solifluxion. La surcharge de la forêt a été incriminée. Or le poids de la biomasse forestière $(1000 \mathrm{t} / \mathrm{ha}$ maxi) est inférieur au poids du manteau neigeux par exemple. L'action mécanique des arbres de haute futaie sur une certaine épaisseur de sol (profondeur d'enracinement) par effet de levier est peut-être un facteur de déséquilibre sur un versant fragile (arbres inclinés dans les terrains en glissement, surchargés de neige ou soumis au vent). Ainsi, l'ONF et la commune de Bourg St Maurice ont fait enlever tous les arbres de futaie situés sur les berges de la Ravoire et les portions de versant en déséquilibre. Sur les terrains exposés au glissement, il est préférable de laisser croître des formations de taillis, à faible développement en hauteur, à troncs courts et souples, à fort pouvoir évapotranspirant (aunes, saules). 